\title{
Analysis of dynamical process with mass distribution of fission fragment in heavy ion reactions
}

\author{
Y. Aritomo ${ }^{1,2}$ \\ 1 Flerov Laboratory of Nuclear Reactions, JINR, Dubna, Moscow Region 141980, Russia \\ 2 Japan Atomic Energy Agency, Tokai, Ibaraki, 319-1195, Japan
}

\begin{abstract}
We analyzed experimental data obtained for the mass distribution of fission fragments in the reactions ${ }^{36} \mathrm{~S}+{ }^{238} \mathrm{U}$ and ${ }^{30} \mathrm{Si}+{ }^{238} \mathrm{U}$ at several incident energies, which were performed by the JAEA group. Using the dynamical model with the Langevin equation, we precisely investigate the incident energy dependence of the mass distribution of fission fragments. We also consider the fine structures in the mass distribution of fission fragments caused by the nuclear structure at a low incident energy. It is explained why the mass distribution of fission fragments has different features in the two reactions. The fusion cross sections are also estimated.
\end{abstract}

\section{Introduction}

Our FLNR theoretical group has recently developed a calculation model that can treat all reaction processes in heavyand superheavy-mass regions, the so called "unified model", which has been applied to several types of reactions [1, 2]. The unified model implies an unified dynamical approach and unified multidimensional potential energy. We then perform a trajectory calculation on the time-dependent unified potential energy surface using the Langevin equation.

Here, we mainly analyze the experimental data obtained for the mass distribution of fission fragments (MDFF) in the reactions ${ }^{36} \mathrm{~S}+{ }^{238} \mathrm{U}$ and ${ }^{30} \mathrm{Si}+{ }^{238} \mathrm{U}$ at several incident energies, which were measured by the JAEA group $[3,4]$. The analysis of the mass distribution of fission fragments is a powerful tool for investigating the mechanism of the reaction in the heavy- and superheavy-mass regions. Using the dynamical model, we precisely investigate the incident energy dependence of mass distribution of fission fragments. This study is the first attempt to treat such experimental data systematically. We also consider the fine structures in the mass distribution of fission fragments caused by the shell structure at a low incident energy. In the reaction ${ }^{30} \mathrm{Si}+{ }^{238} \mathrm{U}$, there are two peaks at approximately $A=$ 90 and 178 at a low incident energy, which do not correspond to nuclei with a closed shell. Using our model, we analyze the data obtained from this reaction in an attempt to understand the origin of these peaks. We also calculate the capture cross section $\sigma_{\text {cap }}$ and the fusion cross section $\sigma_{f u s}$, and compare them with the experimental data.

In section 2, we briefly explain our framework in this study and the model. We show the calculation results of the MDFF in the reaction ${ }^{36} \mathrm{~S}+{ }^{236} \mathrm{U}$ and ${ }^{30} \mathrm{Si}+{ }^{236} \mathrm{U}$ at several incident energies in section 3 . In section 4 , we discuss the reason why the shape of the MDFF for both reactions are different from each other at the low incident energy. In section 5, we present a summary of this study.

\section{Model}

Mainly, we focus our attention on the dynamics of the fusionfission process in superheavy mass region. In the experimental data for the mass and TKE distribution of fission fragments, each reaction type (fusion-fission process, quasifission process, deep-inelastic collision, elastic scattering, etc.) appears simultaneously, and the reactions are coupled with each other. Therefore, we apply an unified dynamical approach and unified multidimensional potential energy, which were proposed by Zagrebaev and Greiner [1, 2]. We perform a trajectory calculation on the potential energy surface. Thus, the whole evolution of the heavy nuclear system can be traced starting from the infinite distance between the projectile and target to the end of each process.

We apply a new method for the calculation of the multidimensional potential energy using the extended two-center shell model [5]. We take into account the time evolution from the diabatic potential $V_{\text {diab }}(q)$ to the adiabatic potential $V_{\text {adiab }}(q)$, here $q$ denotes the collective coordinates of nuclear deformation. The diabatic potential is calculated by the folding procedure with effective nucleon-nucleon interaction $[1,2,5]$. We use the adiabatic potential energy of the nuclear system calculated using the two-center shell model. We connect the diabatic potential and adiabatic potential with a weight function as follows; $V=V_{\text {diab }}(q) f(t)+$ $V_{\text {adiab }}(q)[1-f(t)], f(t)=\exp \left(-\frac{t}{\tau}\right)$. Here, $t$ is the time of interaction and $f(t)$ is a weight function on the relaxation time $\tau$. We use the relaxation time $\tau=10^{-21} \mathrm{sec}$, which was suggested in references [6].

As the coordinates of nuclear deformation, we use the two-center parametrization $[7,8]$ and employ three param- 
eters as follows: $z_{0}$ (distance between two potential centers), $\delta$ (deformation of fragments), and $\alpha$ (mass asymmetry of the colliding nuclei); $\alpha=\left(A_{1}-A_{2}\right) /\left(A_{1}+A_{2}\right)$, where $A_{1}$ and $A_{2}$ denote the mass numbers of the target and the projectile, respectively. The detail is explained in reference [9].

We perform the trajectory calculation on the time-dependent unified potential energy using the Langevin-type equation. The multidimensional Langevin equation is described precisely in reference [1].

The adiabatic potential energy is defined as

$$
\begin{gathered}
V_{\text {adiab }}(q, L, T)=V_{L D}(q)+\frac{\hbar^{2} L(L+1)}{2 I(q)}+V_{S H}(q, T), \\
V_{L D}(q)=E_{S}(q)+E_{C}(q), V_{S H}(q, T)=E_{\text {shell }}^{0}(q) \Phi(T), \\
\Phi(T)=\exp \left(-\frac{E^{*}}{E_{d}}\right)
\end{gathered}
$$

where $I(q)$ is the moment of inertia of a rigid body at deformation $q$. $V_{L D}$ and $V_{S H}$ are the potential energy of the finite-range liquid drop model and the shell correction energy taking into account the temperature dependence. $E_{\text {shell }}^{0}$ is the shell correction energy at $T=0$. The temperature dependent factor $\Phi(T)$ is discussed in reference [10], where $E^{*}$ denotes the excitation energy of the compound nucleus. The shell damping energy $E_{d}$ is chosen as $20 \mathrm{MeV}$, which is given by Ignatyuk et al. $E_{S}$ and $E_{C}$ denote a generalized surface energy and Coulomb energy, respectively. The centrifugal energy arising from the angular momentum $L$ of the rigid body is also considered. The detail is explained in reference [9].

Each trajectory starts from a sufficiently large distance between both nuclei. The capture and fusion cross sections are calculated as follows,

$$
\begin{aligned}
\sigma_{c a p} & =\frac{\pi \hbar^{2}}{2 \mu_{0} E_{c m}} \sum_{\ell=0}^{\infty}(2 \ell+1) T_{\ell}\left(E_{c m}, \ell\right), \\
\sigma_{f u s} & =\frac{\pi \hbar^{2}}{2 \mu_{0} E_{c m}} \sum_{\ell=0}^{\infty}(2 \ell+1) P_{C N}\left(E_{c m}, \ell\right),
\end{aligned}
$$

where $\mu_{0}$ denotes the reduced mass in the entrance channel. $T_{\ell}\left(E_{c m}, \ell\right)$ is the capture probability of the $\ell$-th partial wave. $P_{C N}\left(E_{c m}, \ell\right)$ is the probability of forming a compound nuclei in competition with quasi-fission events. The method used to estimate the probabilities $T_{\ell}\left(E_{c m}, \ell\right)$ and $P_{C N}\left(E_{c m}, \ell\right)$ by a dynamical calculation is explained in the next section.

\section{Incident energy dependence of mass distribution of fission fragments}

\subsection{Reaction of ${ }^{36} \mathrm{~S}+{ }^{238} \mathrm{U}$}

The fragment mass distributions for fission after full momentum transfer were measured by the JAEA group for

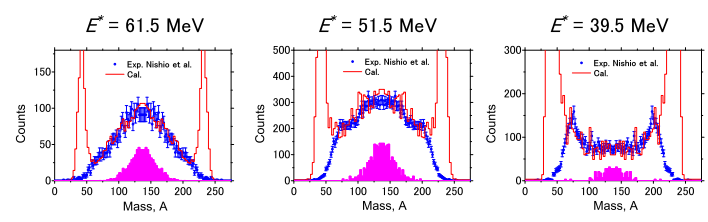

Fig. 1. Mass distributions of fission fragments in the reaction ${ }^{36} \mathrm{~S}+{ }^{238} \mathrm{U}$. Experimental data and calculation results are denoted by circles [3] and histograms, respectively. Fusion-fission events correspond to shaded areas.

the reaction ${ }^{36} \mathrm{~S}+{ }^{238} \mathrm{U}$ at several bombardment energies around the Coulomb barrier [3]. Figure 1 shows the MDFF at $E_{c . m}=176.0\left(E^{*}=61.5\right) \mathrm{MeV}, E_{c . m}=166.0\left(E^{*}=51.5\right) \mathrm{MeV}$ and $E_{c . m}=154.0\left(E^{*}=39.5\right) \mathrm{MeV}$, which are denoted by circles. At the high incident energy, the mass-symmetric fission fragments are dominant. On the other hand, at the low incident energy, the mass-asymmetric fission fragments are dominant. We can observe $\mathrm{Pb}$ fragments, which have a strong nuclear structure.

Since the nuclei of the actinides are prolately deformed, the Coulomb barrier height depends on the orientation of the target nuclei and reactions start from different configurations with the nuclei in contact. The two extreme cases are collisions at poles and equator of the target nucleus. At a low projectile energy, nuclear contact only occurs in polar collisions, whereas at a higher energy, nuclear contact also occurs in equatorial collisions.

As a first approximation, we start the calculation for the spherical-spherical configuration at the high incident energy. Since all configurations are possible at the high incident energy, we use this assumption when considering average configurations. At the low incident energy, we take the initial condition as a collision with the pole of the target ${ }^{238} \mathrm{U}$ nucleus, whose deformation is $\beta_{2}=0.215(\delta \sim 0.2)$.

The calculation results are denoted by histograms in Fig. 1. At the incident energies $E^{*}=61.5 \mathrm{MeV}$ and $E^{*}=$ $51.5 \mathrm{MeV}$, we start the calculation for the spherical-spherical configuration. With the low incident energy, $E^{*}=39.5$ $\mathrm{MeV}$, as the initial condition, we consider the polar collision of the target. The calculations are normalized to agree with experimental data in the mass-symmetric fission region. The trend of the experimental data, i. e., the incident energy dependence of the MDFF, is reproduced by the calculation. However, there is a discrepancy for very asymmetric mass region (above around $A=210$ and below 40 or 50).

The MDFF in experimental data includes only fission events and does not include the elastic and/or nucleontransfer events, or deep inelastic components. In the experiment, fission events can be separately clearly from the elastic and/or nucleons transfer events as we can see in Fig.1(c) of the reference [3]. The mass distribution was obtained by using events entering in the 'Fission'-gate in Fig.1(c). In addition, fission events followed by the nucleontransfer were removed in the off-line analysis, by using the information on the fragment emission angle. That is the experimental spectra include fission events when momentum 

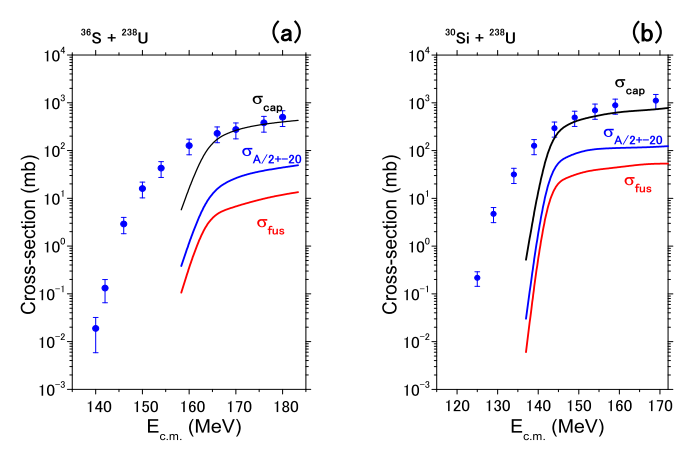

Fig. 2. Excitation function of $\sigma_{c a p}, \sigma_{A / 2 \pm 20}$ and $\sigma_{f u s}$ in the reactions ${ }^{36} \mathrm{~S}+{ }^{238} \mathrm{U}$ (a) and ${ }^{30} \mathrm{Si}+{ }^{238} \mathrm{U}$ (b). Experimental data of $\sigma_{\text {fiss }}$ are denoted by circles [3] and [4], respectively.

of the projectile is fully transferred to the system. However, the calculation was included all processes. Therefore, the calculation results do not agree with the experimental data at very asymmetric mass region.

The calculation and experimental data at the low incident energy show the peak around $A=200$ in the MDFF. It seems that this peak is originated from the shell effects, which R. Kalpakchieva et. al. pointed out [11]. We precisely discuss the mechanism creating this peak in the next section.

In the experiment, the cross sections for the full momentum transfer fission $\left(\sigma_{\text {fiss }}\right)$ are shown in Fig. 2(a) as function of the incident energy [3]. The cross sections are almost equal to those of the projectiles captured inside the Coulomb barrier. The calculation results for the capture cross section $\sigma_{c a p}$ and fusion cross section $\sigma_{f u s}$ are denoted, respectively.

To estimate the cross sections, we start the calculation for the spherical-spherical configuration as a first approximation. The calculation using the Langevin equation is a classical one; therefore, we obtain the cross sections above the Bass barrier region $\left(V_{c . m}^{\text {Bass }}=158.8 \mathrm{MeV}\right)$. We calculate the capture cross section using the procedure in references [12], in which the capture cross sections were derived by counting the events within a frame using a twodimensional matrix of counts as a function of the mass and total kinetic energy. The calculated fusion cross section $\sigma_{f u s}$ is derived from the trajectory crossing the fusion region. We define the fusion region as the inside of the fission barrier in the three-dimensional coordinate space [9]. In Fig. 2(a) the cross section $\sigma_{A / 2 \pm 20}$, which derived from the yield of mass-symmetric fission fragments whose mass number is greater than $A_{C N} / 2-20$ and less than $A_{C N} / 2+20$, is denoted. Here, $A_{C N}$ denotes the mass number of the compound nucleus. The fusion cross section $\sigma_{f u s}$ is one or two order of magnitude smaller than the cross section $\sigma_{A / 2 \pm 20}$. In the MDFF, we also plot the fusion events, which are denoted by shaded areas in Fig. 1. We can see that it is possible to obtain the mass-symmetric fission events in the quasi-fission process [9].

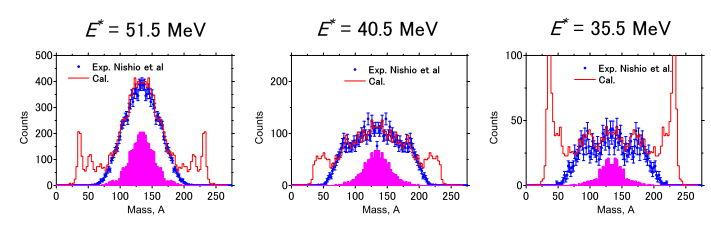

Fig. 3. Mass distributions of fission fragments in the reaction ${ }^{30} \mathrm{Si}+{ }^{238} \mathrm{U}$. Experimental data and calculation results are denoted by circles [4] and histograms, respectively. Fusion-fission events correspond to shaded areas.

\subsection{Reaction of ${ }^{30} \mathrm{Si}+{ }^{238} \mathrm{U}$}

In the MDFF for the reaction ${ }^{36} \mathrm{~S}+{ }^{238} \mathrm{U}$ at the low incident energy, mass-asymmetric fission events are dominant. We can observe a peak near $\mathrm{Pb}$ fragments in Fig. 1. However, in the reaction ${ }^{30} \mathrm{Si}+{ }^{238} \mathrm{U}$ at the low incident energy, the overall tendency and shape of the MDFF are different from those in the reaction ${ }^{36} \mathrm{~S}+{ }^{238} \mathrm{U}$. The MDFF in the reaction ${ }^{30} \mathrm{Si}+{ }^{238} \mathrm{U}$ has also been measured by the JAEA group [4]. In Fig. 3, the experimental data at the incident energies $E_{c . m}=144.0\left(E^{*}=50.5\right) \mathrm{MeV}, E_{c . m}=134.0\left(E^{*}=40.5\right) \mathrm{MeV}$ and $E_{c . m}=129.0\left(E^{*}=35.5\right) \mathrm{MeV}$ are denoted by circles. At the low incident energy, $E^{*}=35.5 \mathrm{MeV}$, mass-symmetric fission events are dominant, and three significant peaks appear at $A \sim 90,134$ and 178. It is not obvious that the peaks at $A \sim 90$ and 178 correspond to nuclei with a strong nuclear structure.

The calculation results are shown in Fig. 3 as histograms, in which we use the same parameters as those in the reaction ${ }^{36} \mathrm{~S}+{ }^{238} \mathrm{U}$. At the incident energies, $E^{*}=51.5$ $\mathrm{MeV}$ and $E^{*}=40.5 \mathrm{MeV}$, we start the calculation for the spherical-spherical configuration. At the low incident energy, $E^{*}=35.5 \mathrm{MeV}$, we take the initial condition as a collision with the pole of the target ${ }^{238} \mathrm{U}$ nucleus. The results at high and low incident energies are in agreement with the tendencies of the experimental data. The calculation results of the capture cross section $\sigma_{\text {cap }}$, fusion cross section $\sigma_{\text {fus }}$ and mass symmetric fission cross section $\sigma_{A / 2 \pm 20}$ are denoted in Fig. 2(b), respectively. We obtain these cross sections performing the calculation for the spherical-spherical configuration as a first approximation. The Bass barrier of this system is $V_{c . m}^{\text {Bass }}=141.1 \mathrm{MeV} . \sigma_{f u s}$ is one or two orders of magnitude smaller than the cross section $\sigma_{A / 2 \pm 20}$, which shows the same tendency as that in the reaction ${ }^{36} \mathrm{~S}$ $+{ }^{238} \mathrm{U}$. We also plot the fusion events, which are denoted by shaded area in Fig. 3. It shows that mass-symmetric fission events come from fusion-fission process and quasifission process.

\section{Analysis of mass distribution of fission fragments at low incident energy}

We investigate the reason why the shapes of the MDFF for both reactions are different from each other at the low incident energy. The shape of the MDFF is affected by the 
landscape of the potential energy surface. Therefore, we investigated the effect of the potential energy surface by calculating the MDFF using the potential energy $V_{L D}$ and $V_{L D}+E_{\text {shell }}^{0}$ for the adiabatic potential energy part. When we use $V_{L D}+E_{\text {shell }}^{0}$, the peaks appear at $A \sim 90$ and 178 in the reaction ${ }^{30} \mathrm{Si}+{ }^{238} \mathrm{U}$ in the MDFF, because the potential energy surface has valleys at $A \sim 90$ and 180 which appear owing to the balance between $V_{L D}$ and $E_{\text {shell }}^{0}$. This is a fine feature of the MDFF. On the other hand, the general features of the MDFF appear to be mainly governed by the landscape of potential energy around the contact point, which is caused by the delicate balance between the diabatic potential and $V_{L D}$ of the adiabatic potential. It determines whether the distribution has a convex or concave shape. The detail is described in reference [13].

\section{Summary}

In this article we analyzed the experimental data obtained for the MDFF in the reactions ${ }^{36} \mathrm{~S}+{ }^{238} \mathrm{U}$ and ${ }^{30} \mathrm{Si}+{ }^{238} \mathrm{U}$ at several incident energies, which were measured by the JAEA group $[3,4]$. We applied an unified model and performed a trajectory calculation. The incident energy dependence of the MDFF was precisely investigated. This study is the first attempt to treat such experimental data systematically. We also discussed the fine structures in the MDFF caused by the shell structure at a low incident energy. The incident energy dependence of experimental data was reproduced by the calculation. At the low incident energy, it is significant that we take the initial condition as a collision with the pole of the target ${ }^{238} \mathrm{U}$ nucleus. We also stress that it is very important to consider the temperature dependence of shell correction energy on the potential energy surface.

The author is grateful to Prof. V.I. Zagrevaev, Prof. W. Greiner, Dr. A.V. Karpov and Prof. F. Hanappe for their helpful suggestions and valuable discussion. The special thanks are deserved to Dr. A.K. Nasirov, Dr. A.S. Denikin and Prof. K. Hagino for their helpful comments, to Dr. K. Nishio, Dr. H. Ikezoe and the JAEA experimental group for providing us the experimental data and their helpful discussion. The diabatic potential and adiabatic potential were calculated using the NRV code [5]. The numerical calculations were carried out on SX8 at YITP in Kyoto Univesiry.

\section{References}

1. V. Zagrebaev and W. Greiner, J. Phys. G 31, 825 (2005).

2. V. Zagrebaev and W. Greiner, J. Phys. G 34, 1 (2007); J. Phys. G 34, 2265 (2007). Phys. Rev. C 78, 034610 (2008); J. Phys. G 35, 125103 (2008).

3. K. Nishio, H. Ikezoe, S. Mitsuoka, I. Nishinaka, Y. Nagame, Y. Watanabe, T. Ohtsuki, K. Hirose, and S. Hofmann, Phys. Rev. C 77, 064607 (2008).

4. K. Nishio, H. Ikezoe, S. Mitsuoka, I, Nishinaka, Y. Watanabe, Y. Nagame, T. Ohtsuki, K. Hirose and S. Hofmann, to be published.
5. V.I. Zagrebaev, A.V. Karpov, Y. Aritomo, M.A. Naumenko, and W. Greiner, Physics of Particles and $\mathrm{Nu}$ clei 38, 469 (2007); NRV codes for driving potential, http://nrv.jinr.ru/nrv/.

6. G.F. Bertsch, Z. Phys. A 289, 103 (1978); W. Cassing and W. Nörenberg, Z. Phys. A 401, 467 (1983); A. DiazTorres, Phys. Rev. C 69, 021603 (2004).

7. J. Maruhn and W. Greiner, Z. Phys. 251, 431 (1972).

8. K. Sato, A. Iwamoto, K. Harada, S. Yamaji, and S. Yoshida, Z. Phys. A 288, 383 (1978).

9. Y. Aritomo and M. Ohta, Nucl. Phys. A 744, 3 (2004).

10. Y. Aritomo, Nucl. Phys. A 780, 222 (2006).

11. R. Kalpakchieva, Yu.Ts. Oganessian, Yu.E. Penionzhkevich, and H. Sodan, Z. Phys. A 283, 253 (1977).

12. W.Q. Shen, J. Albinski, A. Gobbi, S. Gralla, K.D. Hildenbrand, N. Herrmann, J. Kuzminski, W.F.J. Muller, H. Stelzer, J. Toke, B.B. Back, S. Bjornholm, and S.P. Sorensen, Phys. Rev. C 36, 115 (1987).

13. Y. Aritomo, to be published in Phys. Rev. $C$. 